\title{
Design, construction and validation of an all-terrain wheelchair in magnesium alloys
}

XXIV International Conference of the Iberoamerican Society

of Digital Graphics Medellin | Colombia

\author{
Paula Andrea Chacón Cifuentes \\ Universidad Pontificia Bolivariana Sede Central Medellín | Colombia | \\ paula.chacon@upb.edu.co \\ Gustavo Adolfo Sevilla Cadavid \\ Universidad Pontificia Bolivariana Sede Central Medellín | Colombia | \\ gustavo.sevilla@upb.edu.co
}

\author{
Alejandro Alberto Zuleta Gil \\ Universidad Pontificia Bolivariana Sede Central Medellín | Colombia | \\ alejandro.zuleta@upb.edu.co
}

\author{
Andrés Hernando Valencia Escobar \\ Universidad Pontificia Bolivariana Sede Central Medellín | Colombia | \\ andres.valencia@upb.edu.co
}

\begin{abstract}
Magnesium alloys have an excellent strength-to-weight ratio and can be manufactured with a lower environmental impact. Although its application is attractive for wheelchair design, it is little explored. As a result of this work, an all-terrain wheelchair was designed, exhibiting a weight reduction of $32 \%$ compared to commercial products. Users performed usability tests, to evaluate the improvements achieved. This wheelchair can contribute to improving their quality of life, allowing users to save energy and preserve the health of their upper limbs. Finally, an impact was achieved in the local industry, developing capacities to manufacture products in these alloys.
\end{abstract}

Keywords: Light alloys; assistive technology; usability; mobility; sustainability.

\section{INTRODUCCIÓN}

En contextos con terrenos irregulares se requiere un mayor esfuerzo para propulsar una silla de ruedas manual de diseño convencional, por lo que han surgido algunos modelos denominados todoterreno. Estos modelos cuentan con un diseño que les da mayor estabilidad y permiten reducir el esfuerzo en la propulsión manual en este tipo de terrenos. Sin embargo, por ser de mayor tamaño estas sillas de ruedas suelen tener un peso elevado, lo que puede causar afectaciones en los miembros superiores de los usuarios dada la fatiga que se genera con el desplazamiento (Liu et al., 2008; Medola et a., 2014; Rispin \& Wee, 2015). Debido a esto, se propuso diseñar una silla de ruedas todoterreno de propulsión manual, utilizando aleaciones de magnesio, con el fin de obtener un producto liviano y a su vez eficiente en la movilidad por terrenos irregulares.

Las aleaciones de magnesio exhiben unas de las mejores relaciones resistencia-peso dentro de los metales estructurales, lo cual las hace atractivas para este tipo de aplicaciones (Polmear, StJohn, Nie, \& Qian, 2017; You, Huang, Kainer, \& Hort, 2017). Además, estas aleaciones cuentan con otras propiedades como una buena absorción de vibraciones, buena maquinabilidad y cada vez hay mayor disponibilidad de presentaciones comerciales diversas y a un precio competitivo respecto al aluminio
(Anes et al., 2016; Berrio-Betancur et al., 2017). A pesar de esto, su uso en el diseño de sillas de ruedas manuales aún se encuentra poco explorado, lo cual representa una oportunidad para aprovechar sus propiedades en el mejoramiento de estos productos de apoyo.

Por otro lado, las sillas de ruedas todoterreno que se encuentran en el mercado, además de su peso elevado, tienen otros retos por resolver. Uno de los más importantes es su asequibilidad, siendo en su mayoría productos de alto costo y que solo tienen canales de distribución establecidos en unos pocos países, su mayoría en países de ingresos altos (Good Life Medical, 2018; GRIT Freedom Chair, n.d.; Lasher Sport, 2017; Mountain Tirke, 2018; Trekinetic, 2018). Aunque algunas organizaciones sin ánimo de lucro han desarrollado algunos productos de bajo costo que se adaptan a las necesidades cognitivas y financieras de las poblaciones menos favorecidas, estos no atienden en mayor medida las necesidades de rehabilitación física de esta población (Rodrigues Carriel, 2015). Una forma de superar este reto es desarrollar capacidades para el diseño de este tipo de productos en países de ingresos medios y bajos. De esta manera pueden lograrse productos contextualizados en las necesidades locales, a un costo menor y mayores posibilidades de acceder a estos en dichos contextos (World Health Organization, 2011). 
En este trabajo se muestra el proceso de diseño de una silla de ruedas todoterreno en aleaciones de magnesio, que puede fabricarse utilizando tecnología disponible a nivel local. La silla de ruedas diseñada utiliza un mecanismo de propulsión por palancas para aumentar su eficiencia en terrenos irregulares (Flemmer \& Flemmer, 2016). También, utiliza tres puntos de apoyo para lograr una mayor estabilidad y mejorar la maniobrabilidad (Medola et al., 2014). Además, cuenta con algunos parámetros adaptables, para ajustarse a la antropometría del usuario y a su biomecánica.

Finalmente, el diseño propuesto fue evaluado en términos de usabilidad y desempeño técnico. Para evaluar la usabilidad se construyeron prototipos y se desarrollaron pruebas con usuarios en diversas etapas del proyecto. Para la validación técnica se siguieron los protocolos sugeridos en la norma ISO7176 (International Organization for Standardization, 2014, 2015).

\section{METODOLOGÍA}

El proceso de diseño se dividió en tres fases. La primera fase consistió en la búsqueda y análisis de información para la definición de los requerimientos de diseño. Se consultaron estudios médicos alrededor de la movilidad en silla de ruedas y las recomendaciones de las entidades relacionadas con la discapacidad motriz. Se realizó un análisis de los productos ofrecidos en el mercado global, para reconocer las soluciones técnicas existentes. También, se realizaron entrevistas con usuarios y expertos en el campo, con el fin de identificar sus necesidades alrededor de la movilidad en silla de ruedas y contrastarlas con las características de la oferta comercial y las recomendaciones encontradas en la literatura. Se realizaron entrevistas semiestructuradas a tres personas usuarias permanentes de sillas de ruedas, con lesiones de diversa naturaleza y antigüedad (entre 3 y 18 años). Estos participantes tenían características antropométricas y biomecánicas diferentes, lo cual permitió una diversidad de información suficiente para identificar necesidades comunes y necesidades específicas que requerían un ajuste particular de su silla de ruedas. Se realizaron preguntas abiertas que indagaban sobre su percepción alrededor de la adecuación de su silla de ruedas en el marco de las actividades de la vida diaria (AVD) y su nivel de satisfacción con cada uno de los componentes de su silla de ruedas. Cada entrevistado firmó un consentimiento informado y una autorización para el uso de fotografías y videos registrados durante las entrevistas. Partiendo de la información recopilada de las fuentes mencionadas se definieron los requerimientos de diseño, estableciendo las condiciones de ergonomía, desempeño, durabilidad, apariencia, materiales y procesos de fabricación.

En la segunda fase se definió el diseño detallado de la silla de ruedas y se construyeron prototipos funcionales en aleaciones de aluminio y aleaciones de magnesio. Para esto, se comenzó con un proceso de generación y selección de propuestas de diseño. Posteriormente se desarrolló un modelo digital, definiendo las dimensiones, materiales y características formales detalladas que aseguraban el funcionamiento adecuado de la silla de ruedas. También se definieron las relaciones de unión con componentes comerciales como las ruedas, los frenos de disco y demás componentes mecánicos. Con el modelo digital se llevó a cabo un análisis estructural por elementos finitos para evaluar el diseño propuesto. Dicha evaluación se realizó en términos de tres criterios de diseño estructural: resistencia, rigidez y estabilidad (Valencia Escobar, 2007). Se ejecutó un análisis estático lineal utilizando el software Solid Works (Dassault Systèmes, 2016). Posterior al análisis, se realizaron los ajustes requeridos y se generó la información necesaria para la manufactura de los prototipos. El primero fue fabricado en aluminio 6061 T6, buscando poder iniciar la fabricación del de manera más rápida y con un costo menor. De esta manera se pudieron realizar las primeras pruebas de usabilidad que permitieron identificar los ajustes finales. Finalmente, se construyó el prototipo en la aleación de magnesio AZ31B.

En la tercera fase se aplicaron pruebas de usabilidad y pruebas técnicas. Las pruebas de usabilidad se centraron en evaluar las características de eficacia, eficiencia y satisfacción con la participación directa de usuarios. Para evaluar la usabilidad, se realizaron pruebas utilizando los prototipos construidos. En estas participaron los tres usuarios de sillas de ruedas anteriormente entrevistados. Cada participante firmó un consentimiento informado y una autorización para el uso de fotografías y videos registrados durante las validaciones. La prueba consistía en realizar una transferencia de su silla de ruedas al prototipo, seguido de un período de 10 minutos para familiarizarse con su funcionamiento. Luego, se trasladaba a un campo adyacente, el cuál presentaba un terreno con irregularidades como desniveles, raíces de árboles y rocas. En este terreno se realizaba un recorrido de libre elección durante 30 minutos, mientras se registraban las apreciaciones del participante. Al finalizar el recorrido se realizaron preguntas abiertas sobre su percepción alrededor de la estabilidad, el confort, la seguridad y el esfuerzo requerido para maniobrar la silla de ruedas. Los comentarios de los participantes fueron analizados posteriormente para identificar aspectos positivos y aspectos a mejorar en términos de eficacia y satisfacción.

También se realizaron pruebas cuantitativas para evaluar el efecto de la reducción del peso sobre la eficiencia de la silla de ruedas. Para esto, se utilizó una muestra de conveniencia, seleccionando 18 participantes con estatura entre los 1,60 y $1,80 \mathrm{~m}$, un peso máximo de $85 \mathrm{Kg}$ y buen estado de salud. Antes de iniciar las pruebas, cada participante firmó un consentimiento informado y una autorización para el uso de fotografías y videos registrados durante las pruebas. En esta prueba cada participante debía realizar un recorrido de 6 minutos alrededor de un cuadrilátero con lados de $10 \mathrm{~m}$ a una velocidad cómoda autoseleccionada. Al final del recorrido se registró la distancia total que el participante lograba recorrer en el tiempo establecido. Cada participante debía realizar un recorrido con cada uno de los prototipos. Para el análisis, se compararon los tiempos obtenidos con el prototipo de aluminio y el prototipo de magnesio para establecer el efecto de la reducción de peso. Respecto a las pruebas técnicas se evaluó la estabilidad, resistencia estática, resistencia al impacto, resistencia a la caída y resistencia a la fatiga de acuerdo a los protocolos establecidos en la norma ISO7176 (International Organization for Standardization, 2014, 2015). 


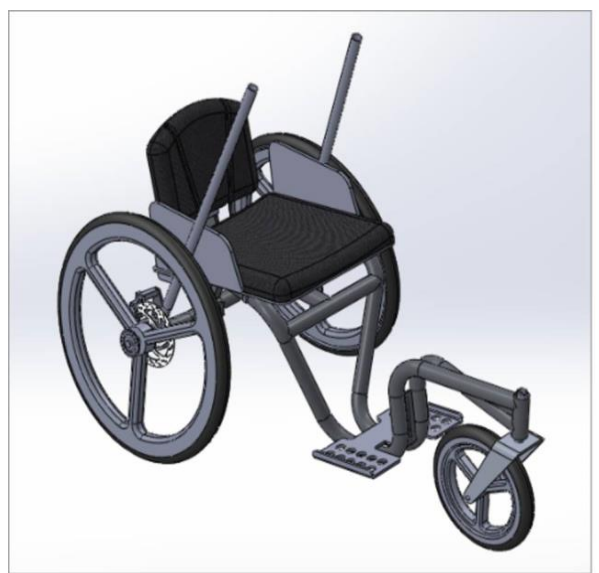

(a)
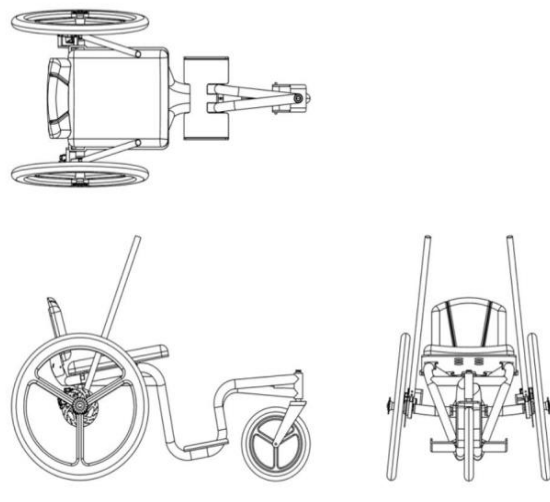

(b)

Figura 1: Modelo digital del diseño final (a) vista en isométrico y (b) vistas ortogonales

\section{RESULTADOS \\ REQUERIMIENTOS DE DISEÑO}

Se obtuvieron los requerimientos de diseño para asegurar el funcionamiento de la silla de ruedas en terrenos irregulares y atender las necesidades de soporte postural, confort y seguridad de los usuarios. Se determinó que la silla estaría dirigida a usuarios de sillas de ruedas que estuviesen en capacidad de autopropulsarse utilizando sus miembros superiores. De esta manera, se seleccionó el mecanismo de propulsión por palancas para obtener una mayor eficiencia en terrenos irregulares. Se decidió utilizar la aleación de magnesio AZ31B para el diseño, debido a su disponibilidad comercial y propiedades más ajustadas a los requerimientos del proyecto. Se estableció que parámetros como el ángulo y la altura del espaldar, la altura de los apoyapiés y la posición del eje de las ruedas posteriores debían poder ajustarse a las particularidades de cada usuario y las diferentes situaciones de uso. El ángulo de avance en el tenedor de la rueda caster permite que esta gire con facilidad al realizar maniobras de viraje y recupere su posición rápidamente.

El ángulo del respaldo se puede adaptar con posibles ángulos de $85^{\circ}, 95^{\circ}$ y $105^{\circ}$. El asiento cuenta con una inclinación de $10^{\circ}$ para aportar seguridad. Sobre una superficie rígida el usuario puede ubicar el cojín de su preferencia. Para mejorar la postura y la distribución de la presión en las piernas es posible graduar la altura de los apoyapiés. El sistema de propulsión por palancas se basa en una adaptación tecnológica que usa sistemas comerciales tipo trinquete como referencia funcional.

Se utilizó tubería de la aleación de magnesio AZ31B, con $38 \mathrm{~mm}$ de diámetro externo y 2,39mm de espesor de pared para la construcción del marco de la silla de ruedas. Esto permitió obtener el comportamiento estructural apropiado y le confiere al producto una apariencia robusta que comunique resistencia.
También se optó por una configuración de tres ruedas, con las dos ruedas posteriores de mayor tamaño y la rueda caster de menor tamaño en posición delantera.

\section{PROPUESTA DE DISEÑO}

Partiendo de los requerimientos y llevando a cabo el proceso descrito en la metodología se obtuvo la propuesta final de diseño (Figura 1). Se seleccionaron ruedas neumáticas comerciales de 24" para las ruedas posteriores y de 12,5" para la rueda delantera. Estas características contribuyen a reducir la resistencia a la rodadura y a mejorar la comodidad del usuario a través de la absorción de vibraciones. El ángulo cámber en las ruedas traseras es de $6^{\circ}$ para proporcionar estabilidad. La posición horizontal de los ejes posteriores puede ajustarse de acuerdo con las necesidades del usuario, para equilibrar la estabilidad del sistema y la biomecánica de las extremidades superiores.

\section{CONSTRUCCIÓN DE PROTOTIPOS}

Con el prototipo en aluminio 6061 T6 (Figura 2) se obtuvo un peso total de la silla de ruedas de $20 \mathrm{Kg}$ y peso de transporte, sin las ruedas traseras, de $13 \mathrm{Kg}$. La fabricación y ensamble del prototipo tomó alrededor de 100 horas de mano de obra y tuvo un costo total de 2072 USD aproximadamente, distribuidos como se muestra en la tabla 1. El prototipo fabricado en magnesio AZ31B (Figura 3) alcanzó un peso total de $15 \mathrm{Kg}$ y un peso de transporte de $8 \mathrm{Kg}$. Para este prototipo el costo fue de 2050 USD aproximadamente, en la tabla 1 puede encontrarse la distribución de dichos costos.

Tabla 1: Costos de fabricación y ensamble de los prototipos construidos.

\begin{tabular}{lll}
\hline Rubro & Prototipo Al & Prototipo Mg \\
\hline Mano de obra & $79 \%$ & $77 \%$ \\
Materia prima & $10 \%$ & $12 \%$ \\
Componentes comerciales & $11 \%$ & $11 \%$ \\
Costo Total (USD) & 2072 & 2050 \\
\hline
\end{tabular}


Con el uso del magnesio AZ31B se alcanzó una reducción de peso del $25 \%$ respecto al prototipo de aluminio, si se comparan los pesos totales. Sin embargo, si se comparan los pesos de transporte, la reducción de peso asciende al $38 \%$. Al comparar el peso total obtenido en el prototipo de magnesio con el peso total reportado de otros modelos todoterreno de tres ruedas, se alcanza una reducción entre $12 \%$ y el $32 \%$ (Figura 4 ).

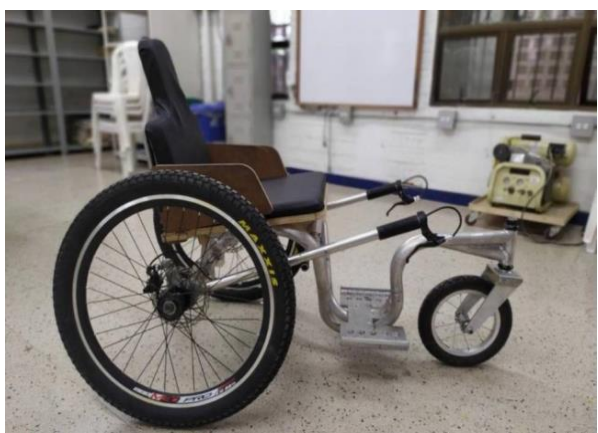

Figura 2: Prototipo en aluminio 6061 T6

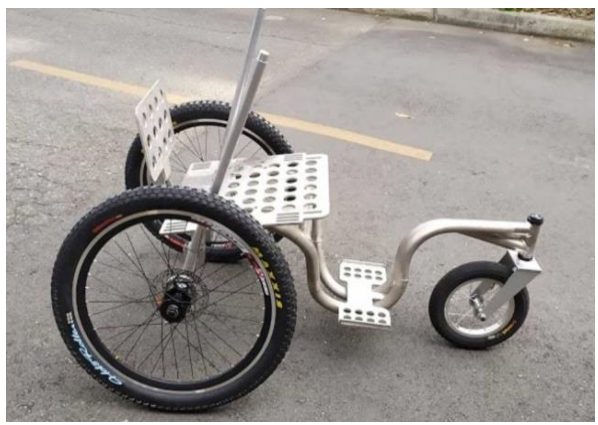

Figura 3: Prototipo en aleación de magnesio AZ31B.

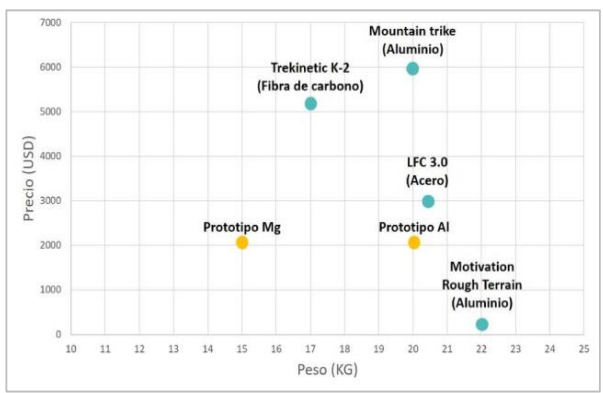

Figura 4: Prototipo en aleación de magnesio AZ31B

Respecto a los costos, puede observarse que la diferencia entre el costo del prototipo de aluminio y el costo del prototipo de magnesio no se encuentra una diferencia considerable. Por otro lado, respecto a los modelos todoterreno de tres ruedas, se encuentra una brecha entre el costo de los prototipos y el precio de venta de los productos comerciales. Esto sugiere que sería posible establecer un precio de venta competitivo, más aún, si se tiene en cuenta que los productos mencionados no se encuentran disponibles a nivel local. Lo anterior implicaría que, para acceder a alguno de dichos productos se tendría que incurrir en un gasto adicional para su importación.

\section{VALIDACIÓN DE LA USABILIDAD}

En las pruebas de usabilidad se pudo confirmar la eficacia del mecanismo de propulsión manual por palancas para desplazarse por terrenos irregulares (Figura 5). Sin embargo, los usuarios percibieron que el avance era corto en comparación con la fuerza realizada, por lo que es necesario explorar el diseño mecánico para aumentar la cantidad de avance que se logra con cada impulsión. Por otra parte, aunque la silla está diseñada para desplazarse en espacios exteriores, se encontró que implementar la posibilidad de desplazarse en reversa sería útil para asegurar la maniobrabilidad en espacios reducidos. En términos de la satisfacción, entendida como la forma en que la silla de ruedas se adapta al usuario, los participantes expresaron que fue fácil aprender a maniobrar la silla. También resaltaron que la posibilidad de personalizar las dimensiones de las interfaces para un mayor ajuste es indispensable para un adecuado posicionamiento y control. Por último, sugirieron la incorporación de correas de seguridad en el asiento y el espaldar para dar soporte a las piernas y al tronco.

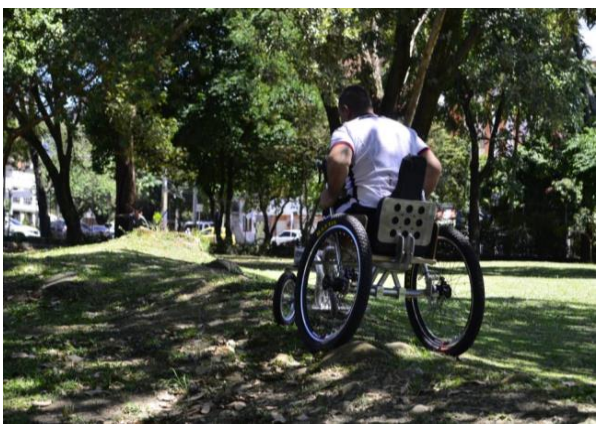

Figura 5: Pruebas de usabilidad en terreno irregular.

En las pruebas alrededor de la eficiencia se pudo observar que los usuarios lograron ahorrar tiempo en sus desplazamientos cuando utilizaron el prototipo fabricado en magnesio. Los valores obtenidos para la distancia recorrida con el prototipo de aluminio en un tiempo de 6 minutos oscilan entre 94 y $220 \mathrm{~m}$, mientras que la distancia recorrida con el prototipo de magnesio en el mismo tiempo oscila entre 100 y $263 \mathrm{~m}$. En promedio, los usuarios recorrieron $152 \mathrm{~m}$ en el prototipo de aluminio, mientras que con el prototipo de magnesio recorrieron $184 \mathrm{~m}$. Con estos datos, puede observarse que con la silla de magnesio se recorre una mayor distancia en el mismo tiempo. Es por esto que, partiendo de la definición de velocidad, se puede inferir que en la silla de magnesio se logra avanzar una misma distancia en un menor tiempo respecto a la silla de aluminio, representando una mejora en la eficiencia de la silla de ruedas.

\section{VALIDACIONES TÉCNICAS}

La norma ISO 7176 establece que al finalizar cada una de las pruebas de resistencia se debe realizar una inspección visual en búsqueda de deformaciones, grietas o fracturas en los componentes de la silla de ruedas y de desajustes entre estos que comprometan su funcionalidad y estabilidad. Después de realizar los protocolos para las 
pruebas de resistencia estática en los apoyapiés, resistencia al impacto en la rueda caster, resistencia a la caída y resistencia a la fatiga, el prototipo no mostró alteraciones visibles y se mantuvo funcional. Debido a lo anterior, se consideró que el prototipo superó satisfactoriamente las condiciones establecidas para las pruebas de resistencia en la norma citada.

Para las pruebas de estabilidad posterior y lateral, la norma no ofrece valores de referencia, sino que determina que se deben registrar los ángulos máximos en los que la silla se mantiene estable (Figura 6). Estos ángulos se registran para la configuración más estable y menos estable de la silla de ruedas.

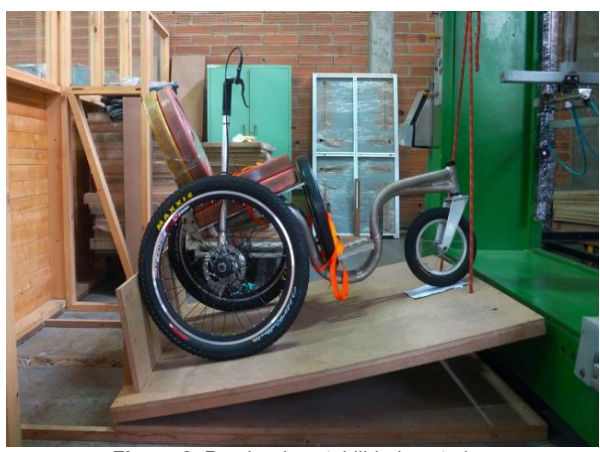

Figura 6: Prueba de estabilidad posterior

Se encontró que los ángulos alcanzados en las pruebas alcanzan valores aceptables, excepto para la posición menos estable y un peso de $100 \mathrm{Kg}$ en la prueba de estabilidad hacia atrás. La configuración menos estable se da cuando el espaldar está reclinado a $105^{\circ}$ y los apoyapiés están en su posición más baja. En esta prueba se alcanzó un ángulo máximo de $4^{\circ}$ o $7 \%$ de inclinación.

\section{DISCUSIÓN}

En primer lugar, el haber logrado un producto más liviano y más eficiente con la aplicación exitosa de las aleaciones de magnesio, muestra cómo la implementación de nuevas tecnologías puede impactar la calidad de vida de las personas. Esta mejora puede contribuir a la preservación de la salud de los miembros superiores los usuarios, especialmente para aquellos que se encuentran en entornos rurales donde las condiciones del terreno exigen un esfuerzo elevado y sobrecargas en esta parte del cuerpo. Por otra parte, un producto con estas características les proporciona una mayor autonomía al facilitarles la movilidad en terrenos irregulares.

En segundo lugar, es importante notar que quedan retos por resolver. La implementación de un mecanismo de reversa y el aumento de la ventaja mecánica del mecanismo de propulsión son pareas que pueden profundizarse en futuras investigaciones. También, es importante considerar que el $47 \%$ del peso de la silla de ruedas diseñada está representado por las ruedas. Si se tiene en cuenta que este es un componente comercial, sería pertinente explorar otras opciones disponibles que permitan reducir el peso total de la silla.
Por otro lado, se hace necesario plantear una modificación para permitir a los usuarios utilizar la configuración menos estable sin comprometer su estabilidad. Esto puede lograrse permitiendo que las ruedas traseras se desplacen hacia atrás del espaldar o que la posición anteroposterior del espaldar pueda modificarse para desplazar la ubicación del centro de masa del sistema hacia adelante. También puede considerarse la instalación de dispositivos antivuelco.

Por último, se destaca el impacto que a través de este proyecto pudo lograrse en la industria local. La fabricación de los prototipos permitió desarrollar capacidades locales para el uso y transformación de aleaciones de magnesio, materiales que pueden representar una opción para disminuir peso en diversas aplicaciones y una oportunidad para utilizar materiales y procesos más sostenibles.

\section{CONCLUSIONES}

Con el prototipo de magnesio se alcanzó una reducción de peso del $25 \%$ respecto al peso total del prototipo de aluminio. Si se comparan los pesos de transporte, se obtuvo una reducción del $39 \%$. Respecto a los modelos comerciales todoterreno de tres ruedas, se logró una reducción entre el $12 \%$ y el $32 \%$. Esta reducción puede incluso incrementarse si se exploran otras alternativas de ruedas que disminuyan el peso total de la silla.

En términos de la eficacia se comprobó la utilidad del mecanismo de palancas para la impulsión manual en terrenos irregulares. Respecto a la satisfacción, se encontró que la silla de ruedas es de fácil comprensión y uso. Además, que el diseño del chasis permite el ensamble de interfaces ajustables a las preferencias del usuario, las cuales pueden modificarse mediante procesos de fabricación menos costosos y más rápidos.

En las pruebas de usabilidad se pudo validar que la reducción de peso alcanzada tuvo un efecto positivo, validando que con la silla de magnesio se recorre una mayor distancia en el mismo tiempo. Esta puede considerarse una mejora en la eficiencia de la silla de ruedas ya que permite al usuario ahorrar tiempo en sus desplazamientos.

Al finalizar las pruebas técnicas bajo la norma ISO 7176-1 y 7176-8 se pudo observar que el prototipo de magnesio superó las pruebas exitosamente, presentando un comportamiento estructural apropiado y condiciones de ajuste aptas para esta aplicación. Para las mejorar las condiciones de estabilidad posterior en la configuración menos estable se pueden implementar soluciones simples como la modificación de la posición del espaldar o la inclusión de dispositivos antivuelco.

Con la fabricación del prototipo de magnesio se logró un impacto positivo en la industria local, ya que ahora pueden encontrarse proveedores con experiencia en maquinado, doblado de tubería y corte por chorro de agua en aleaciones de magnesio. Esto abre la posibilidad de desarrollar otros productos ajustados a las necesidades del contexto local, donde la relación resistencia/peso sea apreciada para proporcionar atributos de movilidad, portabilidad y ahorro energético. 
El costo de los prototipos fue muy similar. Aunque el costo de la materia prima del prototipo de magnesio aumentó un $13 \%$, la diferencia total entre los dos prototipos fue cercana al $1 \%$. De lo anterior puede decirse que para este caso el uso de las aleaciones de magnesio no representa un aumento de costos significativo.

\section{AGRADECIMENTOS}

Los autores agradecen al "Departamento Administrativo de Ciencia, Tecnología e Innovación (COLCIENCIAS)" del Gobierno de Colombia (Beca 1115745-58322), "Centro de Investigación para el Desarrollo y la Innovación (CIDI)" de la Universidad Pontificia Bolivariana a través de la UPB Innova Rad: 747B-03 / 17-35, Universidad de Antioquia, SENA y Universidad de Medellín.

\section{REFERENCIAS}

Anes, V., Lage, Y. E., Vieira, M., Maia, N. M. M., Freitas, M., \& Reis, L. (2016). Torsional and axial damping properties of the AZ31B-F magnesium alloy. Mechanical Systems and Signal Processing, 79, 112-122. https://doi.org/https://doi.org/10.1016/j.ymssp.2016.02.040

Berrio-Betancur, L. F., Echeverry-Rendón, M., Correa-Bedoya, E., Zuleta-Gil, A. A., Robledo-Restrepo, S. M., CastañoGonzalez, J. G., \& Echeverría-Echeverríaa, F. (2017). Development of the magnesium alloy industry in Colombia - an opportunity | Desarrollo de la industria de aleaciones de magnesio en Colombia - una oportunidad. DYNA (Colombia),

84(203). https://doi.org/10.15446/dyna.v84n203.66440

Dassault Systèmes. (2016). Solid Works. Vélizy-Villacoublay, Francia.

Flemmer, C. L., \& Flemmer, R. C. (2016). A review of manual wheelchairs. Disability and Rehabilitation: Assistive Technology, 11(3), 177-187. https://doi.org/10.3109/17483107.2015.1099747

Good Life Medical. (2018). Quickie GT. Retrieved June 10, 2018, from

https://www.goodlifemedical.com.au/products/manualwheelchairs/quickie-gt/

GRIT Freedom Chair. (n.d.). GRIT Freedom Chair - All Terrain Wheelchair. Retrieved November 5, 2019, from https://www.gogrit.us/

International Organization for Standardization. (2014). International Standard ISO 7176-1. Geneva: ISO. https://doi.org/10.1109/IEEESTD.2007.4288250

International Organization for Standardization. International Standard ISO 7176-8. Geneva: ISO.

(2015).

Lasher Sport. (2017). Lasher Sport BT-Mg. Retrieved November 6, 2017, from http://www.lashersport.com/pages/chairs/btmg/btmg.html

Liu, H., Cooper, R. A., Pearlman, J., \& Connor, S. (2008). Evaluation of titanium ultralight manual wheelchairs using ANSI / RESNA standards. Journal of Rehabilitation Research \& Development, 45(9), 1-20. https://doi.org/10.1682/JRRD.2007.12.0204

Medola, F. O., Elui, V. M. C., Santana, C. D. S., \& Fortulan, C. A. (2014). Aspects of Manual Wheelchair Configuration Affecting Mobility: A Review. Journal of Physical Therapy Science, 26, 313-318.

Mountain Tirke. (2018). Mountain trike. Retrieved June 10, 2018, from http://www.mountaintrike.com/products/mountaintrike

Polmear, I., StJohn, D., Nie, J.-F., \& Qian, M. (2017). Light Alloys: Metalurgy of the Light Metals ( $5^{\circ}$ Edición). ButterworthHeinemann.
Rispin, K., \& Wee, J. (2015). Comparison between performances of three types of manual wheelchairs often distributed in low-resource settings. Disability and Rehabilitation: Assistive Technology, 10(4), 316-322.

Rodrigues Carriel, I. R. (2015). Recomendações tecnológicas de projeto para o desenvolvimento de cadeira de rodas de propulsão manual: uma proposta para ampliar o grau de mobilidade dos cadeirantes a partir do design. Universidade de São Paulo.

Trekinetic. (2018). Trekinetic K-2. Retrieved June 10, 2018, from http://www.trekinetic.com/K2.php

Valencia Escobar, A. H. (2007). La estructura: un elemento técnico para el diseño (Primera Ed). Medellín: Editorial Universidad Pontificia Bolivariana.

World Health Organization. (2011). Joint position paper on the provision of mobility devices in less-resourced settings: a step towards implementation of the Convention on the Rights of Persons with Disabilities (CRPD) related to personal mobility. Retrieved from https://www.who.int/disabilities/publications/technology/jpp _final.pdf

You, S., Huang, Y., Kainer, K. U., \& Hort, N. (2017). Recent research and developments on wrought magnesium alloys. Journal of Magnesium and Alloys, 5, 239-253. https://doi.org/10.1016/j.jma.2017.09.001 